\title{
Do Spin State Changes Matter in Organometallic Chemistry?

\author{
A Computational Study.
}

Jose-Luis Carreon-Macedo and Jeremy N. Harvey*

School of Chemistry and Centre for Computational Chemistry, University of Bristol, Cantock's Close,

Bristol BS8 1TS, UK. Email: jeremy.harvey@bristol.ac.uk

\section{Suplementary Information.}

\section{Full details of the BS1, BS2, BSW1 and BSW2 basis sets used for the ab initio calculations.}

Cobalt: the exponents of the polarization functions were optimized at the $\mathrm{CI}$ level for $\mathrm{Co}^{+}\left({ }^{3} \mathrm{~F}\right)$, using the Ahlrichs-TZV (17s,10p,6d)/[6s,3p,3d] basis set as a starting point. Two bases of increasing size were developed. Where required, these basis are simply referred to as "BS1" and "BS2". In the first one, the most diffuse primitive within the contraction scheme was decontracted for the $s, p$, and $d$ functions. Additionally, one diffuse $s$ function $(\alpha=0.02)$ and three (uncontracted) diffuse $p$ functions $(\alpha=0.141308,0.043402,0.018)$ were added. The final contraction was of the form $(18 \mathrm{~s}, 13 \mathrm{p}, 6 \mathrm{~d}, 2 \mathrm{f}, 1 \mathrm{~g}) /[8 \mathrm{~s}, 7 \mathrm{p}, 4 \mathrm{~d}, 2 \mathrm{f}, 1 \mathrm{~g}]$ which included two f functions $\left(\alpha_{1}=4.147168, \alpha_{2}=1.063376\right)$ and one $\mathrm{g}$ function $(\alpha=2.7)$. The second basis set, BS2, was constructed from the previous one. For this we added one $\mathrm{d}$ function $(\alpha=0.1)$ and optimized for $\mathrm{f}\left(\alpha_{1}=6.82, \alpha_{2}=2.20, \alpha_{3}=0.709677\right)$, two g $\left(\alpha_{1}=\right.$ $\left.4.723135, \alpha_{2}=1.431253\right)$ and one h function $(\alpha=3.0)$. The final segmented contraction was of the form $(18 \mathrm{~s}, 13 \mathrm{p}, 7 \mathrm{~d}, 3 \mathrm{f}, 2 \mathrm{~g}, 1 \mathrm{~h}) /[8 \mathrm{~s}, 7 \mathrm{p}, 5 \mathrm{~d}, 3 \mathrm{f}, 2 \mathrm{~g}, 1 \mathrm{~h}]$.

For tungsten we also developed a basis set which was optimized at the CI level for $\mathrm{WH}_{6}$. In this case we used for the metallic atom the Stuttgart/Köln group ECP which uses quasi-relativistic reference data. The corresponding basis set for this potential is a modified segmented contraction of the form $(8 \mathrm{~s} 7 \mathrm{p} 6 \mathrm{~d} 2 \mathrm{f}) /[6 \mathrm{~s} 5 \mathrm{p} 4 \mathrm{~d} 2 \mathrm{f}]$, where the most diffuse $\mathrm{d}$ primitive in the contraction scheme was decontracted and two f functions $\left(\alpha_{1}=0.528205, \alpha_{2}=0.170389\right)$ were included. Another set tried for this potential used three f functions $\left(\alpha_{1}=1.02, \alpha_{2}=0.3, \alpha_{3}=0.088235\right)$ instead of two and one additional $g$ function $(\alpha=0.3)$. We named these basis sets as "BSW1" and "BSW2" respectively. 
Table S1. DFT and CCSD(T) computed energetics $(\mathrm{kcal} / \mathrm{mol})$ of the simplified model ${ }^{1}\left[\mathrm{M}\left(\mathrm{H}_{3}\right)\right] \quad \mathrm{M}=$ $\mathrm{W}\left(\mathrm{NH}_{2}\right)_{3} \mathrm{NH}_{3}($ See Figure S1)

\section{DFT $^{\mathrm{a}} \quad \operatorname{CCSD}(\mathrm{T})^{\mathrm{b}}$}

\begin{tabular}{ccccc}
\hline Model & B3LYP & BP86 & BSW1 & BSW2 \\
\hline $\begin{array}{c}{ }^{3}[\mathrm{M}(\mathrm{H})] \\
+\mathrm{H}_{2}\end{array}$ & 0.0 & 0.0 & 0.0 & 0.0 \\
\hline $\begin{array}{c}1 \mathrm{M}(\mathrm{H})] \\
+\mathrm{H}_{2}\end{array}$ & 24.2 & 23.6 & 24.9 & 24.8 \\
\hline${ }^{1}\left[\mathrm{M}\left(\mathrm{H}_{3}\right)\right]$ & -11.4 & -22.4 & -15.7 & -15.0
\end{tabular}

a) Basis W: LACV3P, N,H: 6-31G*, $\mathrm{H}$ (hydrides):6-31G**

b) Basis W: ECP60MWB + 2f (BS1) or $2 \mathrm{f} 1 \mathrm{~g}$ (BS2), N,H: cc-pVDZ, H(hydrides): cc-pVTZ
Figure S1. Symmetrized $\mathrm{C}_{3 \mathrm{v}}$ simplified model ${ }^{1}\left[\mathrm{M}\left(\mathrm{H}_{3}\right)\right]$

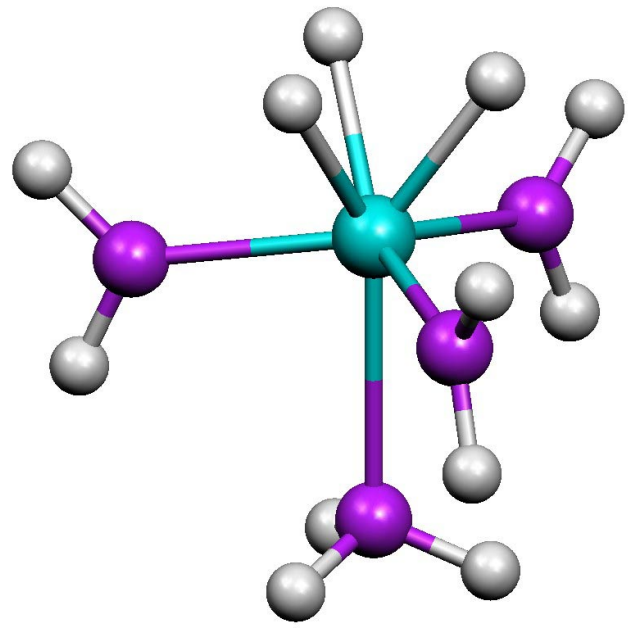

Table S2. Selected geometry parameters of ${ }^{1}\left[\mathrm{CpCoCO}\left(\mathrm{CH}_{3}\right) \mathrm{H}\right]$ for different DFT methods, bond distances $(\bullet)$ and angles $\left({ }^{\circ}\right)$

\begin{tabular}{lllllll}
\hline & B3LYP & B3PW91 & B3PW91* & BPW91 & BP86 & PW91 \\
\hline $\mathrm{C}_{\mathrm{Cp}}-\mathrm{C}_{\mathrm{Cp}}$ & 1.415 & 1.415 & 1.416 & 1.424 & 1.425 & 1.423 \\
$\mathrm{Co}-\mathrm{C}_{\mathrm{Cp}}$ & 2.156 & 2.118 & 2.115 & 2.134 & 2.137 & 2.130 \\
$\mathrm{Co}-\mathrm{CO}$ & 1.734 & 1.717 & 1.711 & 1.714 & 1.715 & 1.710 \\
$\mathrm{C}_{\mathrm{Cp}}-\mathrm{H}_{\mathrm{Cp}}$ & 1.082 & 1.082 & 1.084 & 1.088 & 1.090 & 1.088 \\
$\mathrm{C}-\mathrm{O}$ & 1.151 & 1.152 & 1.156 & 1.169 & 1.170 & 1.168 \\
$\mathrm{Co}-\mathrm{C}_{\mathrm{CH} 3}$ & 1.983 & 1.970 & 1.972 & 1.996 & 1.994 & 1.988 \\
$\mathrm{Co}-\mathrm{H}_{\mathrm{H}}$ & 1.469 & 1.463 & 1.464 & 1.477 & 1.477 & 1.476 \\
$\mathrm{C}_{\mathrm{Cо}}-\mathrm{Co}-\mathrm{C}_{\mathrm{CH} 3}$ & 88.7 & 88.1 & 88.3 & 89.1 & 89.3 & 89.1 \\
$\mathrm{C}_{\mathrm{CH} 3}-\mathrm{Co}_{-}-\mathrm{H}_{\mathrm{H}}$ & 82.4 & 81.5 & 81.3 & 81.0 & 81.2 & 81.0 \\
$\mathrm{H}_{\mathrm{H}} \mathrm{Co}-\mathrm{C}_{\mathrm{CO}}$ & 85.1 & 84.3 & 84.5 & 85.6 & 85.8 & 85.7 \\
\hline
\end{tabular}


Table S3. Some calculated and experimental bond distances $(\bullet)$ and angles $\left({ }^{\circ}\right)$ for ${ }^{1}\left[\mathrm{~W}\left\{\mathrm{~N}_{3} \mathrm{~N}\right\}(\mathrm{H})_{3}\right]$

\begin{tabular}{|c|c|c|c|c|}
\hline & B3LYP & B3LYP $^{a}$ & BP86 & Exp. \\
\hline & 2.026 & 1.996 & 2.026 & $2.000(5)$ \\
\hline \multirow[t]{2}{*}{$\mathrm{W}-\mathrm{N}_{\mathrm{eq}}$} & 2.027 & 1.996 & 2.027 & $2.003(5)$ \\
\hline & 2.033 & 1.996 & 2.032 & $2.013(5)$ \\
\hline \multirow[t]{2}{*}{$\mathrm{W}-\mathrm{N}_{\mathrm{ax}}$} & 2.322 & 2.385 & 2.313 & $2.226(4)$ \\
\hline & 115.6 & 117.6 & 115.5 & $114.6(2)$ \\
\hline \multirow[t]{3}{*}{$\mathrm{N}_{\mathrm{eq}}-\mathrm{W}-\mathrm{N}_{\mathrm{eq}}$} & 116.2 & 117.6 & 116.3 & $116.0(2)$ \\
\hline & 117.7 & 117.6 & 118.0 & $116.9(2)$ \\
\hline & 125.1 & & 124.9 & $123.6(2)$ \\
\hline \multirow[t]{3}{*}{$\mathrm{W}-\mathrm{N}-\mathrm{Si}$} & 127.1 & & 126.6 & $124.0(3)$ \\
\hline & 128.9 & & 128.5 & $124.3(2)$ \\
\hline & 1.686 & 1.690 & 1.701 & 1.57 \\
\hline \multirow[t]{2}{*}{$\mathrm{W}-\mathrm{H}$} & 1.688 & 1.690 & 1.702 & 1.58 \\
\hline & 1.691 & 1.690 & 1.705 & 1.59 \\
\hline
\end{tabular}

a) Symmetrized $\mathrm{C}_{3 \mathrm{v}}$ simplified model ${ }^{1}\left[\mathrm{M}\left(\mathrm{H}_{3}\right)\right]$ (See Figure $\mathrm{S} 1$ )

b) X-ray crystal structure from Dobbs, D. A.; Schrock, R. R.; Davis, W. M. Inorganica Chimica Acta 1997, 263, 171-180. This is Ref. 8 (can change) in the main paper. 
Table S4. Some calculated and experimental bond Table S5 . Comparison of selected bond distances distances $(\AA)$ and angles $\left(^{\circ}\right)$ for ${ }^{1}\left[\mathrm{Tp}^{i-\mathrm{Pr}, \mathrm{Me}} \mathrm{Co}(\mathrm{CO})_{2}\right]$

$(\AA)$ and angles $\left(^{\circ}\right)$ between calculated ${ }^{3}\left[\mathrm{Tp}^{i-}\right.$

\begin{tabular}{lccc}
\hline & B3LYP & BP86 & Exp. $^{\text {a }}$ \\
\hline Co-N & 2.018 & 2.002 & $1.985(8)$ \\
& 2.025 & 2.008 & $2.002(12)$ \\
& 2.156 & 2.121 & $2.087(11)$ \\
\hline Co-CO & 1.757 & 1.731 & $1.696(19)$ \\
& 1.758 & 1.731 & $1.729(15)$ \\
\hline C-O & 1.155 & 1.173 & $1.162(18)$ \\
& 1.156 & 1.174 & $1.179(23)$ \\
\hline N-Co-N & 91.2 & 91.8 & $90.5(4)$ \\
\cline { 3 - 4 } & 91.9 & 91.8 & $91.9(5)$ \\
\hline C-Co-C & 88.7 & 87.0 & $85.0(8)$ \\
\hline Co-C-O & 178.6 & 178.2 & $173.4(16)$ \\
& 178.8 & 178.2 & $177.5(14)$ \\
\hline
\end{tabular}

\begin{tabular}{|c|c|c|}
\hline & ${ }^{3}\left[\operatorname{Tp}^{i \cdot P r, M e} \operatorname{Co}(C O)\right]$ & ${ }^{3}\left[\operatorname{Tp}^{\mathrm{Np}} \mathrm{Co}(\mathrm{CO})\right]$ \\
\hline & BP86 & Exp. $^{\mathrm{a}}$ \\
\hline & 1.551 & $2.055(5)$ \\
\hline Co-N & 1.551 & $2.055(4)$ \\
\hline & 1.559 & $2.010(4)$ \\
\hline $\mathrm{Co}-\mathrm{CO}$ & 1.804 & $1.769(5)$ \\
\hline $\mathrm{C}-\mathrm{O}$ & 1.156 & $1.145(6)$ \\
\hline & 109.1 & $90.6(2)$ \\
\hline & 109.2 & $91.1(2)$ \\
\hline N-Co-N & 110.1 & $93.2(2)$ \\
\hline Co-C-O & 179.1 & $177.8(6)$ \\
\hline
\end{tabular}

a) X-ray crystal structure from Detrich, J. L.; Konecny, R.; Vetter, W. M.; Doren, D.; Rheingold, A. L.; Theopold, K. H. J Am Chem Soc 1996, 118, 1703-1712. This is Ref. 32 (can change) in the main paper.

a) X-ray crystal structure from Detrich, J. L.;

Reinaud, O. M.; Rheingold, A. L.; Theopold, K. H. J. Am. Chem. Soc. 1995, 117, 11745-11748. This is Ref. 10 (can change) in the main paper. 
Table S6.

Coordinates of some species involved in the reaction $\mathrm{CpCo}(\mathrm{CO})+\mathrm{CO}$

${ }^{1}\left[\mathrm{CpCo}(\mathrm{CO})_{2}\right]$

$\begin{array}{rrrr}\text { Co } & -0.0055 & -0.1124 & 0.0000 \\ \mathrm{C} & 1.3987 & 1.4907 & 0.0000 \\ \mathrm{C} & 0.5459 & 1.5460 & 1.1493 \\ \mathrm{C} & -0.8299 & 1.7239 & 0.7078 \\ \mathrm{C} & -0.8299 & 1.7239 & -0.7078 \\ \mathrm{C} & 0.5459 & 1.5460 & -1.1493 \\ \mathrm{H} & 0.8715 & 1.4897 & 2.1890 \\ \mathrm{H} & -1.7015 & 1.8054 & 1.3573 \\ \mathrm{H} & -1.7015 & 1.8054 & -1.3573 \\ \mathrm{H} & 0.8715 & 1.4897 & -2.1890 \\ \mathrm{H} & 2.4816 & 1.3710 & 0.0000 \\ \mathrm{O} & -0.2245 & -2.0857 & 2.1144 \\ \mathrm{C} & -0.1372 & -1.2936 & 1.2561 \\ \mathrm{O} & -0.2245 & -2.0857 & -2.1144 \\ \mathrm{C} & -0.1372 & -1.2936 & -1.2561\end{array}$

\section{${ }^{3}\left[\mathrm{CpCo}(\mathrm{CO})_{2}\right]$}

$\begin{array}{rrrr}\text { Co } & -0.2586 & -0.2278 & 0.0000 \\ \text { C } & 1.2063 & 1.5379 & 0.0000 \\ \text { C } & 0.3738 & 1.6731 & 1.1606 \\ \text { C } & -0.9789 & 1.7698 & 0.7216 \\ \text { C } & -0.9789 & 1.7698 & -0.7216 \\ \text { C } & 0.3738 & 1.6731 & -1.1606 \\ \text { H } & 0.7096 & 1.6206 & 2.1973 \\ \text { H } & -1.8576 & 1.8651 & 1.3619 \\ \text { H } & -1.8576 & 1.8651 & -1.3619 \\ \text { H } & 0.7096 & 1.6206 & -2.1973 \\ \text { H } & 2.2923 & 1.4341 & 0.0000 \\ \text { O } & 0.4062 & -2.0183 & 2.3000 \\ \text { C } & 0.0957 & -1.3153 & 1.4234 \\ \text { O } & 0.4062 & -2.0183 & -2.3000 \\ \text { C } & 0.0957 & -1.3153 & -1.4234\end{array}$

$\operatorname{MECP}(\mathrm{CpCo}(\mathrm{CO})+\mathrm{CO})$

$\begin{array}{rrrr}\text { Co } & -0.2013 & 0.8380 & 0.0942 \\ \text { C } & -1.8881 & 2.4386 & 0.0686 \\ \text { C } & -1.0630 & 2.7147 & -1.0750 \\ \text { C } & 0.2538 & 2.9574 & -0.6048 \\ \text { C } & 0.2259 & 2.9435 & 0.8447 \\ \text { C } & -1.1067 & 2.6773 & 1.2525 \\ \text { H } & -1.3694 & 2.6371 & -2.1194 \\ \text { H } & 1.1291 & 3.1775 & -1.2202 \\ \text { H } & 1.0753 & 3.1494 & 1.4993 \\ \text { H } & -1.4584 & 2.5762 & 2.2808 \\ \text { H } & -2.9538 & 2.2040 & 0.0454 \\ \text { O } & -0.8970 & -0.9178 & -2.2467 \\ \text { C } & -0.5679 & -0.2373 & -1.3602 \\ \text { O } & -0.6883 & -0.8984 & 2.5047 \\ \text { C } & -0.4447 & -0.2290 & 1.5827\end{array}$




\section{Table S7 .}

\section{Coordinates of some species involved in the reaction $\mathrm{CpCo}(\mathrm{CO})+\mathrm{CH}_{4}$}

\section{${ }^{1}\left[\mathrm{CpCoCO}\left(\mathrm{CH}_{3}\right) \mathrm{H}\right]$}

$\begin{array}{rrrr}\mathrm{C} & -1.6407 & 4.6372 & 1.9849 \\ \mathrm{C} & -1.5206 & 3.1988 & 1.8893 \\ \mathrm{C} & -0.8422 & 2.8875 & 0.6732 \\ \mathrm{C} & -0.5176 & 4.1291 & 0.0155 \\ \mathrm{C} & -1.0334 & 5.2022 & 0.8260 \\ \mathrm{H} & -1.8677 & 2.4782 & 2.6308 \\ \mathrm{H} & -0.5714 & 1.8902 & 0.3264 \\ \mathrm{H} & 0.0176 & 4.2344 & -0.9281 \\ \mathrm{H} & -0.9246 & 6.2667 & 0.6161 \\ \mathrm{H} & -2.1087 & 5.1882 & 2.8011 \\ \mathrm{O} & 1.4040 & 3.5929 & 4.5527 \\ \mathrm{C} & 0.9845 & 3.7998 & 3.4799 \\ \mathrm{CO} & 0.4191 & 4.0753 & 1.8844 \\ \mathrm{H} & 1.5739 & 3.2627 & 1.4501 \\ \mathrm{C} & 1.7628 & 5.5349 & 1.6866 \\ \mathrm{H} & 1.3564 & 6.4345 & 2.1798 \\ \mathrm{H} & 2.7350 & 5.2742 & 2.1317 \\ \mathrm{H} & 1.8934 & 5.7097 & 0.6063\end{array}$

\section{TS $\left(\mathrm{CpCo}(\mathrm{CO})+\mathrm{CH}_{4}\right)$}

$\begin{array}{rrrr}\text { Co } & -1.9923 & 0.6635 & 0.4006 \\ \mathrm{C} & -0.0142 & 0.2963 & 0.8636 \\ \mathrm{H} & -0.1499 & 0.5603 & 1.9264 \\ \mathrm{H} & -0.8509 & 0.0814 & -0.3682 \\ \mathrm{H} & 0.7029 & 0.9930 & 0.3996 \\ \mathrm{H} & 0.3836 & -0.7299 & 0.8129 \\ \mathrm{O} & -2.8837 & -1.9939 & 1.1117 \\ \mathrm{C} & -2.5203 & -0.9122 & 0.8351 \\ \mathrm{C} & -3.7968 & 1.6293 & 0.5101 \\ \mathrm{C} & -3.3064 & 1.7448 & -0.8468 \\ \mathrm{C} & -2.0624 & 2.4317 & -0.7808 \\ \mathrm{C} & -1.8134 & 2.7896 & 0.6035 \\ \mathrm{C} & -2.8982 & 2.3337 & 1.3982 \\ \mathrm{H} & -3.7795 & 1.3374 & -1.7401 \\ \mathrm{H} & -1.3974 & 2.6432 & -1.6193 \\ \mathrm{H} & -0.9210 & 3.3004 & 0.9695 \\ \mathrm{H} & -3.0135 & 2.4485 & 2.4760 \\ \mathrm{H} & -4.7276 & 1.1419 & 0.8054\end{array}$

\section{${ }^{1}\left[\mathrm{CpCoCO} \bullet \mathrm{CH}_{4}\right]$}

$\begin{array}{rrrr}\text { Co } & -2.0940 & 0.6522 & 0.4180 \\ \mathrm{C} & -0.0710 & -0.2185 & -0.2945 \\ \mathrm{H} & 0.0785 & 0.4897 & 0.5423 \\ \mathrm{H} & -1.1638 & -0.4328 & -0.6049 \\ \mathrm{H} & 0.4246 & 0.1647 & -1.1982 \\ \mathrm{H} & 0.3223 & -1.2016 & 0.0015 \\ \mathrm{O} & -2.5596 & -1.3634 & 2.4455 \\ \mathrm{C} & -2.3654 & -0.5441 & 1.6221 \\ \mathrm{C} & -3.9038 & 1.4142 & -0.2909 \\ \mathrm{C} & -2.9203 & 1.7384 & -1.2538 \\ \mathrm{C} & -1.8936 & 2.5302 & -0.5884 \\ \mathrm{C} & -2.2651 & 2.7567 & 0.7657 \\ \mathrm{C} & -3.4714 & 1.9928 & 0.9831 \\ \mathrm{H} & -2.9061 & 1.4278 & -2.2994 \\ \mathrm{H} & -0.9785 & 2.8902 & -1.0648 \\ \mathrm{H} & -1.7223 & 3.3439 & 1.5060 \\ \mathrm{H} & -4.0254 & 1.9304 & 1.9213 \\ \mathrm{H} & -4.8048 & 0.8213 & -0.4498\end{array}$

$\operatorname{MECP}\left(\mathrm{CpCo}(\mathrm{CO})+\mathrm{CH}_{4}\right)$

$\begin{array}{rrrr}\mathrm{Co} & -1.9375 & 0.6292 & 0.5263 \\ \mathrm{C} & 0.5031 & 0.1257 & 0.5682 \\ \mathrm{H} & -0.0411 & 0.4709 & 1.4779 \\ \mathrm{H} & -0.1514 & 0.0956 & -0.3388 \\ \mathrm{H} & 1.3272 & 0.8283 & 0.3696 \\ \mathrm{H} & 0.8688 & -0.8948 & 0.7539 \\ \mathrm{O} & -2.9499 & -2.0473 & 1.1109 \\ \mathrm{C} & -2.4853 & -0.9909 & 0.8862 \\ \mathrm{C} & -3.7856 & 1.6164 & 0.4782 \\ \mathrm{C} & -3.2238 & 1.6619 & -0.8560 \\ \mathrm{C} & -1.9995 & 2.3851 & -0.7775 \\ \mathrm{C} & -1.8061 & 2.7998 & 0.5935 \\ \mathrm{C} & -2.9198 & 2.3488 & 1.3675 \\ \mathrm{H} & -3.6575 & 1.2126 & -1.7498 \\ \mathrm{H} & -1.3109 & 2.5743 & -1.6033 \\ \mathrm{H} & -0.9503 & 3.3633 & 0.9723 \\ \mathrm{H} & -3.0841 & 2.5199 & 2.4314 \\ \mathrm{H} & -4.7223 & 1.1304 & 0.7570\end{array}$


Table S8.

Coordinates of some species involved in the reaction $\mathrm{Tp}^{i-\mathrm{Pr}, \mathrm{Me}} \mathrm{CoCO}+\mathrm{CO}$

${ }^{1}\left[\mathrm{Tp}^{\mathrm{i}-\mathrm{Pr}, \mathrm{Me}} \mathrm{Co}(\mathrm{CO})_{2}\right]$

\begin{tabular}{|c|c|c|c|}
\hline Co & -0.7639 & 0.0871 & -0.4363 \\
\hline $\mathrm{C}$ & -1.1082 & 1.1528 & 2.4763 \\
\hline C & -0.5953 & 0.7958 & 3.7448 \\
\hline C & 0.2778 & -0.2731 & 3.5300 \\
\hline $\mathrm{N}$ & 0.2713 & -0.5487 & 2.1892 \\
\hline $\mathrm{N}$ & -0.5797 & 0.3199 & 1.5437 \\
\hline $\mathrm{H}$ & -0.8215 & 1.2658 & 4.7014 \\
\hline $\mathrm{C}$ & -1.1902 & -3.0875 & -0.8355 \\
\hline C & -0.6068 & -4.2758 & -0.3298 \\
\hline C & 0.3862 & -3.8694 & 0.5660 \\
\hline $\mathrm{N}$ & 0.3788 & -2.5001 & 0.5801 \\
\hline $\mathrm{N}$ & -0.5784 & -2.0198 & -0.2729 \\
\hline $\mathrm{H}$ & -0.8704 & -5.3031 & -0.5812 \\
\hline $\mathrm{N}$ & 1.9601 & -0.5322 & 0.3518 \\
\hline C & 3.2914 & -0.2326 & 0.2450 \\
\hline C & 3.4120 & 0.8442 & -0.6373 \\
\hline C & 2.0979 & 1.1795 & -1.0383 \\
\hline $\mathrm{N}$ & 1.2291 & 0.3277 & -0.4369 \\
\hline $\mathrm{H}$ & 4.3372 & 1.3321 & -0.9421 \\
\hline B & 1.2170 & -1.4659 & 1.3542 \\
\hline $\mathrm{H}$ & 2.0116 & -2.0136 & 2.0795 \\
\hline $\mathrm{C}$ & -2.0329 & 2.2978 & 2.1380 \\
\hline $\mathrm{H}$ & -2.0998 & 2.3430 & 1.0355 \\
\hline C & 1.1117 & -1.0340 & 4.5178 \\
\hline $\mathrm{H}$ & 0.8971 & -2.1172 & 4.4892 \\
\hline $\mathrm{H}$ & 2.1933 & -0.9096 & 4.3286 \\
\hline $\mathrm{H}$ & 0.8993 & -0.6699 & 5.5365 \\
\hline C & -3.4500 & 2.0758 & 2.7108 \\
\hline $\mathrm{H}$ & -3.4241 & 2.0170 & 3.8146 \\
\hline $\mathrm{H}$ & -4.1153 & 2.9125 & 2.4303 \\
\hline $\mathrm{H}$ & -3.8947 & 1.1411 & 2.3292 \\
\hline $\mathrm{C}$ & -1.4396 & 3.6401 & 2.6237 \\
\hline $\mathrm{H}$ & -1.3401 & 3.6585 & 3.7246 \\
\hline $\mathrm{H}$ & -0.4398 & 3.8136 & 2.1889 \\
\hline $\mathrm{H}$ & -2.0971 & 4.4784 & 2.3295 \\
\hline C & 1.3270 & -4.6935 & 1.3951 \\
\hline $\mathrm{H}$ & 2.3845 & -4.4833 & 1.1521 \\
\hline $\mathrm{H}$ & 1.1976 & -4.5075 & 2.4769 \\
\hline $\mathrm{H}$ & 1.1425 & -5.7647 & 1.2102 \\
\hline $\mathrm{C}$ & -2.3079 & -2.9362 & -1.8396 \\
\hline $\mathrm{H}$ & -2.5022 & -1.8522 & -1.9383 \\
\hline C & -1.8954 & -3.4757 & -3.2276 \\
\hline $\mathrm{H}$ & -1.6781 & -4.5589 & -3.1823 \\
\hline $\mathrm{H}$ & -2.7091 & -3.3228 & -3.9603 \\
\hline $\mathrm{H}$ & -0.9922 & -2.9629 & -3.6017 \\
\hline $\mathrm{C}$ & -3.6058 & -3.6099 & -1.3420 \\
\hline $\mathrm{H}$ & -3.4654 & -4.7000 & -1.2199 \\
\hline $\mathrm{H}$ & -3.9213 & -3.1973 & -0.3678 \\
\hline $\mathrm{H}$ & -4.4257 & -3.4535 & -2.0666 \\
\hline C & 4.3607 & -0.9802 & 0.9853 \\
\hline $\mathrm{H}$ & 5.3501 & -0.5900 & 0.6951 \\
\hline $\mathrm{H}$ & 4.2583 & -0.8733 & 2.0804 \\
\hline $\mathrm{H}$ & 4.3387 & -2.0613 & 0.7588 \\
\hline $\mathrm{C}$ & 1.6582 & 2.3166 & -1.9295 \\
\hline $\mathrm{H}$ & 0.5529 & 2.3390 & -1.8991 \\
\hline $\mathrm{C}$ & 2.1658 & 3.6693 & -1.3807 \\
\hline $\mathrm{H}$ & 3.2705 & 3.7124 & -1.3798 \\
\hline
\end{tabular}

$\begin{array}{ll}4.5001 & -2.0088 \\ 3.8349 & -0.3460 \\ 2.1052 & -3.3919 \\ 2.0713 & -3.4633 \\ 1.1619 & -3.8019 \\ 2.9347 & -4.0291 \\ 0.5226 & -3.3025 \\ 0.3683 & -2.1416 \\ 0.5512 & -0.3481 \\ 0.3851 & -0.3786\end{array}$

\section{${ }^{3}\left[\mathrm{Tp}^{i-\mathrm{Pr}, \mathrm{Me}} \mathrm{Co}(\mathrm{CO})_{2}\right]$}

$\begin{array}{rrrr}\mathrm{Co} & -0.8368 & 0.1933 & -0.4974 \\ \mathrm{C} & -1.2065 & 1.1038 & 2.6598 \\ \mathrm{C} & -0.6874 & 0.6887 & 3.9099 \\ \mathrm{C} & 0.2663 & -0.2925 & 3.6233 \\ \mathrm{~N} & 0.3025 & -0.4413 & 2.2623 \\ \mathrm{~N} & -0.5965 & 0.4109 & 1.6690 \\ \mathrm{H} & -0.9632 & 1.0530 & 4.8994 \\ \mathrm{C} & -1.2445 & -2.9480 & -0.8671 \\ \mathrm{C} & -0.6552 & -4.1220 & -0.3428 \\ \mathrm{C} & 0.3294 & -3.6951 & 0.5509 \\ \mathrm{~N} & 0.3191 & -2.3248 & 0.5527 \\ \mathrm{~N} & -0.6430 & -1.8659 & -0.3176 \\ \mathrm{H} & -0.9102 & -5.1545 & -0.5807 \\ \mathrm{~N} & 2.0013 & -0.4224 & 0.4030 \\ \mathrm{C} & 3.3510 & -0.2333 & 0.2651 \\ \mathrm{C} & 3.5354 & 0.7703 & -0.6911 \\ \mathrm{C} & 2.2380 & 1.1570 & -1.1049 \\ \mathrm{~N} & 1.3185 & 0.4241 & -0.4339 \\ \mathrm{H} & 4.4900 & 1.1662 & -1.0371 \\ \mathrm{~B} & 1.2040 & -1.3373 & 1.3703 \\ \mathrm{H} & 1.9676 & -1.9681 & 2.0609 \\ \mathrm{C} & -2.2572 & 2.1533 & 2.3762 \\ \mathrm{H} & -2.3427 & 2.2340 & 1.2753 \\ \mathrm{C} & 1.1274 & -1.0860 & 4.5621 \\ \mathrm{H} & 0.9520 & -2.1727 & 4.4631 \\ \mathrm{H} & 2.2042 & -0.9125 & 4.3841 \\ \mathrm{H} & 0.9028 & -0.7986 & 5.6025 \\ \mathrm{C} & -3.6347 & 1.7377 & 2.9403 \\ \mathrm{H} & -3.5942 & 1.6341 & 4.0403 \\ \mathrm{H} & -4.4007 & 2.4972 & 2.6981 \\ \mathrm{H} & -3.9637 & 0.7713 & 2.5211 \\ \mathrm{C} & -1.8289 & 3.5359 & 2.9153 \\ \mathrm{H} & -1.7222 & 3.5163 & 4.0155 \\ \mathrm{H} & -0.8605 & 3.8480 & 2.4865 \\ \mathrm{H} & -2.5854 & 4.3012 & 2.6625 \\ \mathrm{C} & 1.2629 & -4.5197 & 1.3886 \\ \mathrm{H} & 2.3226 & -4.3199 & 1.1482 \\ \mathrm{H} & 1.1315 & -4.3273 & 2.4686 \\ \mathrm{H} & 1.0685 & -5.5898 & 1.2071 \\ \mathrm{C} & -2.3679 & -2.8394 & -1.8714 \\ \mathrm{H} & -2.5381 & -1.7630 & -2.0570 \\ \mathrm{C} & -1.9842 & -3.5014 & -3.2138 \\ \mathrm{H} & -1.7962 & -4.5828 & -3.0816 \\ \mathrm{H} & -2.8020 & -3.3878 & -3.9488 \\ \mathrm{H} & -3.6765 & -3.0475 & -3.6376 \\ \mathrm{H} & -4.511 & -1.3102 \\ \mathrm{H} & -1.0965\end{array}$

$\begin{array}{rrrr}\mathrm{H} & -3.9769 & -2.9410 & -0.3732 \\ \mathrm{H} & -4.4985 & -3.3308 & -2.0410 \\ \mathrm{C} & 4.3753 & -1.0097 & 1.0401 \\ \mathrm{H} & 5.3868 & -0.7006 & 0.7289 \\ \mathrm{H} & 4.2863 & -0.8419 & 2.1287 \\ \mathrm{H} & 4.2833 & -2.0975 & 0.8694 \\ \mathrm{C} & 1.8383 & 2.2141 & -2.1091 \\ \mathrm{H} & 0.7326 & 2.2688 & -2.0929 \\ \mathrm{C} & 2.3799 & 3.6035 & -1.7075 \\ \mathrm{H} & 3.4853 & 3.6122 & -1.7025 \\ \mathrm{H} & 2.0389 & 4.3732 & -2.4240 \\ \mathrm{H} & 2.0340 & 3.8892 & -0.6986 \\ \mathrm{C} & 2.2823 & 1.8328 & -3.5392 \\ \mathrm{H} & 3.3834 & 1.7568 & -3.6024 \\ \mathrm{H} & 1.8575 & 0.8609 & -3.8439 \\ \mathrm{H} & 1.9513 & 2.5966 & -4.2666 \\ \mathrm{O} & -0.9922 & 0.3451 & -3.5083 \\ \mathrm{C} & -0.8563 & 0.3244 & -2.3492 \\ \mathrm{O} & -3.8487 & 0.2653 & -0.3220 \\ \mathrm{C} & -2.6823 & 0.3008 & -0.3209\end{array}$




\section{$\operatorname{MECP}\left(\mathrm{Tp}^{i-\mathrm{Pr}, \mathrm{Me}} \mathrm{CoCO}+\mathrm{CO}\right)$}

\begin{tabular}{|c|c|c|c|}
\hline Co & -0.8285 & 0.2024 & -0.4641 \\
\hline C & -1.2078 & 1.1043 & 2.6506 \\
\hline $\mathrm{C}$ & -0.6897 & 0.6880 & 3.9002 \\
\hline C & 0.2631 & -0.2947 & 3.6158 \\
\hline $\mathrm{N}$ & 0.3000 & -0.4451 & 2.2556 \\
\hline $\mathrm{N}$ & -0.5976 & 0.4082 & 1.6605 \\
\hline $\mathrm{H}$ & -0.9639 & 1.0553 & 4.8887 \\
\hline C & -1.2418 & -2.9481 & -0.8646 \\
\hline C & -0.6546 & -4.1248 & -0.3425 \\
\hline C & 0.3306 & -3.7023 & 0.5521 \\
\hline $\mathrm{N}$ & 0.3228 & -2.3324 & 0.5553 \\
\hline $\mathrm{N}$ & -0.6389 & -1.8679 & -0.3110 \\
\hline $\mathrm{H}$ & -0.9117 & -5.1566 & -0.5824 \\
\hline $\mathrm{N}$ & 1.9928 & -0.4235 & 0.4015 \\
\hline $\mathrm{C}$ & 3.3413 & -0.2342 & 0.2619 \\
\hline $\mathrm{C}$ & 3.5238 & 0.7693 & -0.6944 \\
\hline $\mathrm{C}$ & 2.2264 & 1.1580 & -1.1058 \\
\hline $\mathrm{N}$ & 1.3073 & 0.4248 & -0.4314 \\
\hline $\mathrm{H}$ & 4.4780 & 1.1679 & -1.0376 \\
\hline B & 1.2025 & -1.3425 & 1.3685 \\
\hline $\mathrm{H}$ & 1.9709 & -1.9649 & 2.0611 \\
\hline $\mathrm{C}$ & -2.2571 & 2.1555 & 2.3722 \\
\hline $\mathrm{H}$ & -2.3446 & 2.2392 & 1.2719 \\
\hline C & 1.1258 & -1.0844 & 4.5569 \\
\hline $\mathrm{H}$ & 0.9523 & -2.1716 & 4.4613 \\
\hline $\mathrm{H}$ & 2.2026 & -0.9096 & 4.3800 \\
\hline $\mathrm{H}$ & 0.8998 & -0.7942 & 5.5962 \\
\hline $\mathrm{C}$ & -3.6331 & 1.7395 & 2.9394 \\
\hline $\mathrm{H}$ & -3.5907 & 1.6363 & 4.0394 \\
\hline $\mathrm{H}$ & -4.3988 & 2.4994 & 2.6984 \\
\hline $\mathrm{H}$ & -3.9631 & 0.7733 & 2.5207 \\
\hline $\mathrm{C}$ & -1.8267 & 3.5363 & 2.9152 \\
\hline $\mathrm{H}$ & -1.7206 & 3.5149 & 4.0155 \\
\hline $\mathrm{H}$ & -0.8584 & 3.8493 & 2.4871 \\
\hline $\mathrm{H}$ & -2.5831 & 4.3021 & 2.6636 \\
\hline $\mathrm{C}$ & 1.2651 & -4.5243 & 1.3906 \\
\hline $\mathrm{H}$ & 2.3242 & -4.3217 & 1.1498 \\
\hline $\mathrm{H}$ & 1.1331 & -4.3294 & 2.4702 \\
\hline $\mathrm{H}$ & 1.0731 & -5.5951 & 1.2113 \\
\hline $\mathrm{C}$ & -2.3651 & -2.8372 & -1.8693 \\
\hline $\mathrm{H}$ & -2.5356 & -1.7613 & -2.0551 \\
\hline $\mathrm{C}$ & -1.9824 & -3.4990 & -3.2123 \\
\hline $\mathrm{H}$ & -1.7950 & -4.5807 & -3.0809 \\
\hline $\mathrm{H}$ & -2.8006 & -3.3845 & -3.9466 \\
\hline $\mathrm{H}$ & -1.0701 & -3.0452 & -3.6366 \\
\hline C & -3.6743 & -3.4386 & -1.3092 \\
\hline $\mathrm{H}$ & -3.5536 & -4.5168 & -1.0961 \\
\hline $\mathrm{H}$ & -3.9751 & -2.9383 & -0.3725 \\
\hline $\mathrm{H}$ & -4.4954 & -3.3272 & -2.0408 \\
\hline C & 4.3674 & -1.0073 & 1.0379 \\
\hline $\mathrm{H}$ & 5.3776 & -0.6951 & 0.7256 \\
\hline $\mathrm{H}$ & 4.2791 & -0.8385 & 2.1265 \\
\hline $\mathrm{H}$ & 4.2785 & -2.0956 & 0.8685 \\
\hline $\mathrm{C}$ & 1.8339 & 2.2172 & -2.1099 \\
\hline $\mathrm{H}$ & 0.7286 & 2.2761 & -2.0970 \\
\hline C & 2.3808 & 3.6045 & -1.7064 \\
\hline $\mathrm{H}$ & 3.4864 & 3.6110 & -1.7015 \\
\hline $\mathrm{H}$ & 2.0419 & 4.3756 & -2.4223 \\
\hline $\mathrm{H}$ & 2.0354 & 3.8905 & -0.6975 \\
\hline C & 2.2825 & 1.8350 & -3.5386 \\
\hline $\mathrm{H}$ & 3.3838 & 1.7590 & -3.5995 \\
\hline $\mathrm{H}$ & 1.8582 & 0.8632 & -3.8440 \\
\hline $\mathrm{H}$ & 1.9537 & 2.5988 & -4.2670 \\
\hline 0 & -0.9956 & 0.3481 & -3.4537 \\
\hline
\end{tabular}

$\begin{array}{llll}\text { C } & -0.8592 & 0.3524 & -2.2933 \\ \mathrm{O} & -3.8154 & 0.2800 & -0.3363 \\ \mathrm{C} & -2.6479 & 0.3247 & -0.3214\end{array}$


Table S9.

Coordinates of some species involved in the reaction $\left[\mathrm{N}_{3} \mathrm{~N}\right] \mathrm{WH}+\mathrm{H}_{2}$

${ }^{1}\left[\left\{\mathrm{~N}_{3} \mathrm{~N}\right\} \mathrm{W}\right](\mathrm{H})_{3}$

\begin{tabular}{|c|c|c|c|}
\hline W & 0.0111 & 0.0298 & -0.0026 \\
\hline $\mathrm{H}$ & 0.9912 & 0.3008 & 1.3626 \\
\hline $\mathrm{N}$ & -0.0536 & 0.0674 & -2.3145 \\
\hline $\mathrm{N}$ & 0.8531 & 1.8390 & -0.3843 \\
\hline $\mathrm{N}$ & 1.1677 & -1.5780 & -0.4311 \\
\hline $\mathrm{N}$ & -1.9779 & -0.1829 & -0.3296 \\
\hline $\mathrm{C}$ & -1.4741 & 0.3241 & -2.6981 \\
\hline $\mathrm{H}$ & -1.6480 & 0.0292 & -3.7547 \\
\hline $\mathrm{H}$ & -1.6458 & 1.4101 & -2.6050 \\
\hline $\mathrm{C}$ & 0.4193 & -1.2751 & -2.7703 \\
\hline $\mathrm{H}$ & -0.4353 & -1.9684 & -2.6848 \\
\hline $\mathrm{H}$ & 0.7326 & -1.2322 & -3.8349 \\
\hline $\mathrm{C}$ & 0.8535 & 1.1695 & -2.7564 \\
\hline $\mathrm{H}$ & 1.8848 & 0.7777 & -2.7263 \\
\hline $\mathrm{H}$ & 0.6180 & 1.4670 & -3.8003 \\
\hline $\mathrm{C}$ & 1.5442 & -1.7619 & -1.8519 \\
\hline $\mathrm{H}$ & 2.4924 & -1.2294 & -2.0851 \\
\hline $\mathrm{H}$ & 1.7291 & -2.8281 & -2.0932 \\
\hline $\mathrm{C}$ & 0.7404 & 2.3371 & -1.7731 \\
\hline $\mathrm{H}$ & 1.5403 & 3.0634 & -2.0155 \\
\hline $\mathrm{H}$ & -0.2192 & 2.8781 & -1.9286 \\
\hline $\mathrm{C}$ & -2.4063 & -0.4084 & -1.7301 \\
\hline $\mathrm{H}$ & -2.4381 & -1.4945 & -1.9668 \\
\hline $\mathrm{H}$ & -3.4338 & -0.0293 & -1.9 \\
\hline $\mathrm{Si}$ & 1.6529 & 2.8766 & 0.8397 \\
\hline $\mathrm{Si}$ & -3.3128 & -0.1693 & 572 \\
\hline $\mathrm{Si}$ & 1.7399 & -2.8207 & 0.7256 \\
\hline $\mathrm{C}$ & 2.2217 & -2.1238 & 2.4 \\
\hline $\mathrm{H}$ & 1.3528 & -1.7031 & 2.9492 \\
\hline $\mathrm{H}$ & 2.6626 & -2.9298 & 3.0358 \\
\hline $\mathrm{H}$ & 2.9725 & -1.3199 & 2.3089 \\
\hline $\mathrm{C}$ & 0.3872 & -4.1370 & 0.9320 \\
\hline $\mathrm{H}$ & -0.5254 & -3.6714 & 1.3470 \\
\hline $\mathrm{H}$ & 0.1223 & -4.5980 & -0.0385 \\
\hline $\mathrm{H}$ & 0.7091 & -4.9446 & 1.6171 \\
\hline $\mathrm{C}$ & 3.3013 & -3.6554 & 0.0141 \\
\hline $\mathrm{H}$ & 3.6983 & -4.3758 & 0.7550 \\
\hline $\mathrm{H}$ & 3.1144 & -4.2152 & -0.9211 \\
\hline $\mathrm{H}$ & 4.0956 & -2.9112 & -0.1856 \\
\hline $\mathrm{C}$ & 0.5376 & 3.1991 & 2.3353 \\
\hline $\mathrm{H}$ & 0.3178 & 2.2588 & 2.8690 \\
\hline $\mathrm{H}$ & 1.0266 & 3.9009 & 3.0386 \\
\hline $\mathrm{H}$ & -0.4254 & 3.6409 & 2.0185 \\
\hline $\mathrm{C}$ & 2.0550 & 4.5699 & 0.0555 \\
\hline $\mathrm{H}$ & 1.1567 & 5.0650 & -0.3591 \\
\hline $\mathrm{H}$ & 2.4685 & 5.2340 & 0.8389 \\
\hline $\mathrm{H}$ & 2.8121 & 4.5012 & -0.7485 \\
\hline $\mathrm{C}$ & 3.2968 & 2.1172 & 1.4063 \\
\hline $\mathrm{H}$ & 3.8256 & 2.7995 & 2.0998 \\
\hline $\mathrm{H}$ & 3.1227 & 1.1577 & 1.9242 \\
\hline $\mathrm{H}$ & 3.9605 & 1.9225 & 0.5427 \\
\hline $\mathrm{C}$ & -4.1002 & 1.5596 & 0.8552 \\
\hline $\mathrm{H}$ & -3.3605 & 2.3091 & 1.1923 \\
\hline $\mathrm{H}$ & -4.4385 & 1.8477 & -0.1582 \\
\hline$T$ & -4.9753 & 1.6082 & 1.531 \\
\hline
\end{tabular}

$\begin{array}{rrrr}\mathrm{C} & -2.7969 & -0.6183 & 2.6288 \\ \mathrm{H} & -2.1301 & 0.1417 & 3.0689 \\ \mathrm{H} & -3.7021 & -0.7056 & 3.2615 \\ \mathrm{H} & -2.2611 & -1.5839 & 2.6489 \\ \mathrm{C} & -4.6184 & -1.4506 & 0.3283 \\ \mathrm{H} & -5.4503 & -1.4630 & 1.0585 \\ \mathrm{H} & -5.0533 & -1.2388 & -0.6658 \\ \mathrm{H} & -4.1830 & -2.4676 & 0.3004 \\ \mathrm{H} & -0.1925 & -0.9671 & 1.3650 \\ \mathrm{H} & -0.6935 & 0.6901 & 1.3977\end{array}$


TS

$\left(\left[\mathrm{N}_{3} \mathrm{~N}\right] \mathrm{WH}+\mathrm{H}_{2}\right)$

\begin{tabular}{|c|c|c|c|}
\hline $\mathrm{W}$ & 0.1337 & -0.1741 & 0.0777 \\
\hline $\mathrm{H}$ & 0.5682 & -0.9683 & 1.5001 \\
\hline . & 0.4298 & -0.2413 & -2.4186 \\
\hline $\mathrm{N}$ & -1.1520 & -1.5686 & -0.5537 \\
\hline $\mathrm{N}$ & 0.6934 & 1.6534 & -0.4940 \\
\hline $\mathrm{N}$ & 2.0588 & -0.9800 & -0.2154 \\
\hline C & 1.7446 & -0.9124 & -2.6515 \\
\hline $\mathrm{H}$ & 2.1633 & -0.6013 & -3.6322 \\
\hline $\mathrm{H}$ & 1.5749 & -2.0016 & -2.6837 \\
\hline C & 0.4354 & 1.1730 & -2.8845 \\
\hline $\mathrm{H}$ & 1.4609 & 1.4276 & -3.2045 \\
\hline $\mathrm{H}$ & -0.2316 & 1.2989 & -3.7593 \\
\hline C & -0.7028 & -1.0755 & -2.9012 \\
\hline $\mathrm{H}$ & -1.6012 & -0.4361 & -2.9471 \\
\hline $\mathrm{H}$ & -0.5008 & -1.4831 & -3.9161 \\
\hline C & 0.0246 & 2.1109 & -1.7260 \\
\hline $\mathrm{H}$ & -1.0732 & 2.0830 & -1.5863 \\
\hline $\mathrm{H}$ & 0.2982 & 3.1527 & -1.9911 \\
\hline C & -0.9456 & -2.1938 & -1.8695 \\
\hline $\mathrm{H}$ & -1.8237 & -2.7910 & -2.1849 \\
\hline $\mathrm{H}$ & -0.0792 & -2.8874 & -1.8467 \\
\hline C & 2.6977 & -0.6133 & -1.4905 \\
\hline $\mathrm{H}$ & 2.9416 & 0.4736 & -1.4871 \\
\hline $\mathrm{H}$ & 3.6503 & -1.1529 & -1.6798 \\
\hline $\mathrm{Si}$ & -2.7556 & -1.7680 & 0.2392 \\
\hline Si & 3.0131 & -2.1190 & 0.7707 \\
\hline $\mathrm{Si}$ & 1.6144 & 2.8435 & 0.4844 \\
\hline $\mathrm{C}$ & 0.3786 & 4.0619 & 1.2453 \\
\hline $\mathrm{H}$ & -0.3531 & 3.5092 & 1.8621 \\
\hline $\mathrm{H}$ & 0.8941 & 4.7988 & 1.8904 \\
\hline $\mathrm{H}$ & -0.1801 & 4.6186 & 0.4704 \\
\hline $\mathrm{C}$ & 2.5917 & 1.9617 & 1.8370 \\
\hline $\mathrm{H}$ & 1.9202 & 1.4960 & 2.5789 \\
\hline $\mathrm{H}$ & 3.2225 & 1.1636 & 1.4081 \\
\hline $\mathrm{H}$ & 3.2456 & 2.6858 & 2.3606 \\
\hline $\mathrm{C}$ & 2.8281 & 3.7822 & -0.6406 \\
\hline $\mathrm{H}$ & 3.3436 & 4.5730 & -0.0623 \\
\hline $\mathrm{H}$ & 3.6007 & 3.1010 & -1.0437 \\
\hline $\mathrm{H}$ & 2.3242 & 4.2729 & -1.4941 \\
\hline $\mathrm{C}$ & -2.6878 & -1.3993 & 2.0934 \\
\hline $\mathrm{H}$ & -2.5813 & -0.3203 & 2.2919 \\
\hline $\mathrm{H}$ & -3.6177 & -1.7624 & 2.5744 \\
\hline $\mathrm{H}$ & -1.8326 & -1.9163 & 2.5663 \\
\hline $\mathrm{C}$ & -3.2131 & -3.6126 & 0.0762 \\
\hline $\mathrm{H}$ & -2.4302 & -4.2467 & 0.5330 \\
\hline $\mathrm{H}$ & -4.1598 & -3.8033 & 0.6178 \\
\hline $\mathrm{H}$ & -3.3563 & -3.9456 & -0.9683 \\
\hline $\mathrm{C}$ & -4.0860 & -0.7202 & -0.6146 \\
\hline $\mathrm{H}$ & -5.0688 & -0.8638 & -0.1258 \\
\hline $\mathrm{H}$ & -3.8154 & 0.3484 & -0.5467 \\
\hline $\mathrm{H}$ & -4.1979 & -0.9887 & -1.6820 \\
\hline $\mathrm{C}$ & 3.0643 & -3.8090 & -0.1096 \\
\hline $\mathrm{H}$ & 2.0459 & -4.2360 & -0.1773 \\
\hline $\mathrm{H}$ & 3.4709 & -3.7307 & -1.1359 \\
\hline $\mathrm{H}$ & 3.6986 & -4.5251 & 0.4479 \\
\hline $\mathrm{C}$ & 2.3411 & -2.3942 & 2.5200 \\
\hline $\mathrm{H}$ & 1.3484 & -2.8772 & 2.5155 \\
\hline $\mathrm{H}$ & 3.0438 & -3.0532 & 3.0684 \\
\hline
\end{tabular}

$\begin{array}{rrrr}\mathrm{H} & 2.2531 & -1.4458 & 3.0794 \\ \mathrm{C} & 4.8103 & -1.5062 & 0.9599 \\ \mathrm{H} & 5.4124 & -2.2672 & 1.4935 \\ \mathrm{H} & 5.3044 & -1.3117 & -0.0101 \\ \mathrm{H} & 4.8487 & -0.5728 & 1.5526 \\ \mathrm{H} & -0.1673 & 0.5978 & 1.5672 \\ \mathrm{H} & -1.4660 & 0.6074 & 0.2901\end{array}$


Intermediate $\left(\left[\mathrm{N}_{3} \mathrm{~N}\right] \mathrm{WH}+\mathrm{H}_{2}\right)$

\begin{tabular}{|c|c|c|c|}
\hline & 0.0000 & 0.0000 & . \\
\hline $\mathrm{H}$ & 0.0000 & 0.0000 & 1.7189 \\
\hline & 0.9070 & 0.0000 & -2.2304 \\
\hline $\mathrm{N}$ & 0.2704 & -1.8557 & -0.6672 \\
\hline $\mathrm{N}$ & 0.0844 & 1.9177 & -0.5592 \\
\hline $\mathrm{N}$ & 2.0802 & -0.0187 & 0.3628 \\
\hline C & 2.3479 & -0.3691 & -2.0742 \\
\hline $\mathrm{H}$ & 2.9167 & -0.0919 & -2.9882 \\
\hline $\mathrm{H}$ & 2.4074 & -1.4623 & -1.9451 \\
\hline C & 0.7462 & 1.3701 & -2.8184 \\
\hline $\mathrm{H}$ & 1.7328 & 1.8640 & -2.7915 \\
\hline & 0.4306 & 1.3009 & -3.8786 \\
\hline C & 0.1267 & -1.0328 & -2.9601 \\
\hline $\mathrm{H}$ & 0.8644 & -0.5973 & -3.1646 \\
\hline $\mathrm{H}$ & 0.6109 & -1.3007 & -3.9249 \\
\hline C & 0.2440 & 2.1899 & -1.9647 \\
\hline $\mathrm{H}$ & 1.2851 & 1.8758 & -2.1765 \\
\hline F & -0.1438 & 3.2629 & -2.2309 \\
\hline C & -0.0355 & -2.2685 & -2.0623 \\
\hline $\mathrm{H}$ & -0.8683 & -2.8776 & -2.4726 \\
\hline $\mathrm{H}$ & $0 . \varepsilon$ & -2. & -2.1139 \\
\hline C & 2.9054 & 0.3048 & -0.8147 \\
\hline $\mathrm{H}$ & 2.9346 & 1.4058 & -0.9778 \\
\hline $\mathrm{H}$ & 3.9646 & -0.0131 & -0.7056 \\
\hline & -1.3060 & -3.0186 & 0.2467 \\
\hline$i$ & 58 & -0. & 1.7385 \\
\hline $\mathrm{Si}$ & 7 & 3. & 0.4301 \\
\hline C & 0.8 & 4.5 & 0.3408 \\
\hline $\mathrm{H}$ & 1.79 & 4.0 & 0.7542 \\
\hline F & -0.65 & 5.4581 & 0.9372 \\
\hline $\mathrm{H}$ & -1.1004 & 4.8767 & -0.6926 \\
\hline C & 08 & 2.8 & 2.2347 \\
\hline F & 0.0 & 2. & 2 . \\
\hline & 1.7 & 2.1 & 2.3314 \\
\hline & 1.1044 & 3.8020 & 2.8098 \\
\hline C & 2.1252 & 4.1759 & -0.2872 \\
\hline $\mathrm{H}$ & 2.3144 & 5.1 & 0.2374 \\
\hline $\mathrm{H}$ & & 3.5 & -0.1606 \\
\hline $\mathrm{H}$ & 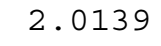 & 4. & -1 \\
\hline c & 1.25 & -2.7 & 2.1137 \\
\hline $\mathrm{H}$ & 1.63 & -1.7 & 2.3832 \\
\hline $\mathrm{H}$ & -1.9 & -3.4737 & 2.5854 \\
\hline I & -0.2 & -2.8 & 2.5372 \\
\hline$C$ & -0.6 & -4.7 & -0.087 \\
\hline & & -4 . & $0.24 \varepsilon$ \\
\hline $\mathrm{F}$ & -1.18 & -5.5055 & 0.4759 \\
\hline $\mathrm{H}$ & -0.6386 & -5.0314 & -1.1561 \\
\hline C & -3.0833 & -2.9105 & -0.4038 \\
\hline$H$ & -3.7321 & -3.6537 & 0.0983 \\
\hline $\mathrm{H}$ & -3.48 & -1.9010 & -0.207 \\
\hline & -3.1 & $-3 . c$ & -1.493 \\
\hline c & 3.74 & -2.3265 & 1.318 \\
\hline $\mathrm{H}$ & 2.9226 & -3.0537 & 1.162 \\
\hline $\mathrm{H}$ & 4.3520 & -2.3026 & 0.395 \\
\hline $\mathrm{H}$ & 4.3850 & -2.7029 & 2.1 \\
\hline C & 2.0419 & -0.7542 & $3.347 \varepsilon$ \\
\hline
\end{tabular}

$\begin{array}{rrrr}\mathrm{H} & 1.2074 & -1.4706 & 3.2626 \\ \mathrm{H} & 2.7195 & -1.1040 & 4.1515 \\ \mathrm{H} & 1.6139 & 0.2164 & 3.6544 \\ \mathrm{C} & 4.5013 & 0.5544 & 2.0888 \\ \mathrm{H} & 5.1183 & 0.1470 & 2.9132 \\ \mathrm{H} & 5.1621 & 0.6802 & 1.2105 \\ \mathrm{H} & 4.1526 & 1.5592 & 2.3941 \\ \mathrm{H} & -1.5667 & 0.0976 & 0.7213 \\ \mathrm{H} & -1.4275 & 0.2310 & -0.9675\end{array}$




\section{$\operatorname{MECP}\left(\left[\mathrm{N}_{3} \mathrm{~N}\right] \mathrm{WH}+\mathrm{H}_{2}\right)$}

\begin{tabular}{|c|c|c|c|}
\hline W & 0.4274 & 0.0191 & 0.1485 \\
\hline $\mathrm{H}$ & 0.1579 & -0.0888 & 1.8689 \\
\hline $\mathrm{N}$ & 0.6900 & -0.0405 & -2.2068 \\
\hline $\mathrm{N}$ & 0.7767 & 1.9702 & -0.3112 \\
\hline $\mathrm{N}$ & 1.2781 & -1.8077 & -0.1756 \\
\hline $\mathrm{N}$ & -1.4489 & -0.3250 & -0.4256 \\
\hline $\mathrm{C}$ & -0.6943 & 0.0692 & -2.7539 \\
\hline $\mathrm{H}$ & -0.7363 & -0.2904 & -3.8044 \\
\hline $\mathrm{H}$ & -0.9767 & 1.1353 & -2.7410 \\
\hline C & 1.3531 & -1.3289 & -2.5763 \\
\hline $\mathrm{H}$ & 0.5609 & -2.0599 & -2.8137 \\
\hline $\mathrm{H}$ & 1.9854 & -1.1941 & -3.4795 \\
\hline $\mathrm{C}$ & 1.5383 & 1.1398 & -2.5257 \\
\hline $\mathrm{H}$ & 2.5689 & 0.8743 & -2.2315 \\
\hline $\mathrm{H}$ & 1.5288 & 1.3604 & -3.6169 \\
\hline $\mathrm{C}$ & 2.1468 & -1.8471 & -1.3629 \\
\hline $\mathrm{H}$ & 3.0510 & -1.2148 & -1.2100 \\
\hline $\mathrm{H}$ & 2.5196 & -2.8690 & -1.5896 \\
\hline $\mathrm{C}$ & 1.0676 & 2.3505 & -1.7110 \\
\hline $\mathrm{H}$ & 1.8614 & 3.1265 & -1.7764 \\
\hline $\mathrm{H}$ & 0.1696 & 2.7978 & -2.1933 \\
\hline $\mathrm{C}$ & -1.6563 & -0.7083 & -1.8456 \\
\hline $\mathrm{H}$ & -1.4951 & -1.8012 & -1.9728 \\
\hline $\mathrm{H}$ & -2.6922 & -0.5036 & -2.1878 \\
\hline $\mathrm{Si}$ & 0.6175 & 3.3885 & 0.7602 \\
\hline $\mathrm{Si}$ & -2.9598 & -0.1267 & 0.5159 \\
\hline $\mathrm{Si}$ & 0.9550 & -3.3534 & 0.6384 \\
\hline $\mathrm{C}$ & 2.5625 & -4.0963 & 1.3353 \\
\hline $\mathrm{H}$ & 2.9785 & -3.4445 & 2.1260 \\
\hline $\mathrm{H}$ & 2.3644 & -5.0900 & 1.7819 \\
\hline $\mathrm{H}$ & 3.3386 & -4.2254 & 0.5579 \\
\hline $\mathrm{C}$ & -0.2792 & -3.1343 & 2.0490 \\
\hline $\mathrm{H}$ & 0.0704 & -2.3769 & 2.7719 \\
\hline $\mathrm{H}$ & -1.2559 & -2.7970 & 1.6603 \\
\hline $\mathrm{H}$ & -0.4271 & -4.0947 & 2.5795 \\
\hline $\mathrm{C}$ & 0.2182 & -4.5704 & -0.6306 \\
\hline $\mathrm{H}$ & 0.0380 & -5.5627 & -0.1745 \\
\hline $\mathrm{H}$ & -0.7507 & -4.1858 & -1.0019 \\
\hline $\mathrm{H}$ & 0.8813 & -4.7204 & -1.5038 \\
\hline $\mathrm{C}$ & -0.2011 & 2.9799 & 2.4115 \\
\hline $\mathrm{H}$ & 0.2921 & 2.1337 & 2.9193 \\
\hline $\mathrm{H}$ & -0.1548 & 3.8689 & 3.0705 \\
\hline $\mathrm{H}$ & -1.2636 & 2.7143 & 2.2703 \\
\hline $\mathrm{C}$ & -0.4588 & 4.6988 & -0.1100 \\
\hline $\mathrm{H}$ & -1.4412 & 4.2731 & -0.3900 \\
\hline $\mathrm{H}$ & -0.6440 & 5.5484 & 0.5752 \\
\hline $\mathrm{H}$ & 0.0097 & 5.1035 & -1.0262 \\
\hline $\mathrm{C}$ & 2.3247 & 4.1517 & 1.1118 \\
\hline $\mathrm{H}$ & 2.2081 & 5.0840 & 1.6982 \\
\hline $\mathrm{H}$ & 2.9506 & 3.4559 & 1.7009 \\
\hline $\mathrm{H}$ & 2.8750 & 4.4035 & 0.1861 \\
\hline $\mathrm{C}$ & -3.8227 & 1.4786 & -0.0308 \\
\hline $\mathrm{H}$ & -3.2146 & 2.3608 & 0.2433 \\
\hline $\mathrm{H}$ & -3.9838 & 1.5062 & -1.1251 \\
\hline $\mathrm{H}$ & -4.8111 & 1.5785 & 0.4575 \\
\hline $\mathrm{C}$ & -2.7081 & -0.0879 & 2.3867 \\
\hline $\mathrm{H}$ & -2.0256 & 0.7168 & 2.7034 \\
\hline $\mathrm{H}$ & -3.6911 & 0.0686 & 2.8734 \\
\hline $\mathrm{H}$ & -2.2858 & -1.0384 & 2.7555 \\
\hline
\end{tabular}

$\begin{array}{rrrr}\mathrm{C} & -4.1167 & -1.5878 & 0.1239 \\ \mathrm{H} & -5.0608 & -1.4839 & 0.6924 \\ \mathrm{H} & -4.3793 & -1.6496 & -0.9483 \\ \mathrm{H} & -3.6521 & -2.5478 & 0.4175 \\ \mathrm{H} & 2.5472 & 0.2954 & 0.6396 \\ \mathrm{H} & 2.1481 & 0.2517 & 1.3247\end{array}$


S-13 